This item was submitted to Loughborough's Research Repository by the author.

Items in Figshare are protected by copyright, with all rights reserved, unless otherwise indicated.

\title{
Meanings of sitting in the context of chronic disease: Critical reflection on sedentary behaviour, health, choice and enjoyment
}

PLEASE CITE THE PUBLISHED VERSION

https://doi.org/10.1080/2159676X.2019.1595105

\section{PUBLISHER}

(C) Informa UK Limited, trading as Taylor \& Francis Group

\section{VERSION}

AM (Accepted Manuscript)

\section{PUBLISHER STATEMENT}

This is an Accepted Manuscript of an article published by Taylor \& Francis in Qualitative Research in Sport, Exercise and Health on 03 Apr 2019, available online: https://doi.org/10.1080/2159676X.2019.1595105

\section{LICENCE}

CC BY-NC-ND 4.0

\section{REPOSITORY RECORD}

Weedon, Amie, Paula Saukko, John Downey, Mark W. Orme, Dale Esliger, Sally Singh, and Lauren Sherar. 2019. "Meanings of Sitting in the Context of Chronic Disease: Critical Reflection on Sedentary Behaviour, Health, Choice and Enjoyment”. Loughborough University. https://hdl.handle.net/2134/37317. 


\section{Meanings of sitting in the context of chronic disease: \\ Critical reflection on sedentary behaviour, health, choice and enjoyment ${ }^{1}$}

Abstract

Reducing sedentary behaviour or sitting is a new public health focus. Emerging research has, however, found that sedentary activities may be associated with health and mental health benefits for older adults. This article reports findings of the qualitative arm of a feasibility trial to reduce sedentary behaviour among patients with Chronic Obstructive Pulmonary Disease (COPD). From interviews $(n=21)$ conducted prior to the intervention we identified three themes: (i) participants sat to enable them to perform activities, such as housework, (ii) sitting, such as watching TV or fishing, was experienced as enjoyable, and (iii) the most ill participants experienced sitting in terms of sadness, as the only thing they could do. Our observations draw attention to three issues. First, our participants did not always sit out of choice, they had to rest between activities and sat due to breathlessness and mournfulness. Second, the intrinsic value of enjoyment associated with sedentary activities comes into sharp relief in the context of progressive chronic disease, which makes it increasingly difficult to enjoy any activity or life. Third, trials, predicated on trying out a pre-defined solution, are particularly challenging for mixed methods qualitative research seeking to trouble categories, such as choice, health and enjoyment. In conclusion we concur with research that has highlighted that sedentary activities may also have benefits, however, we would make a stronger case for appreciating alternative values, such as enjoyment of life, rather than just health, when appropriate, in research and in practice.

Key words: COPD; sedentary behaviour, qualitative research methods

\footnotetext{
${ }^{1}$ Weedon, A., Saukko, P. Downey, J. Orme, M., Singh, S., Esliger, D. \& Sherar, L. (forthcoming) Meanings of sitting in the context of chronic disease: Critical reflection on sedentary behaviour, health, choice and enjoyment, Qualitative Research in Sport, Exercise and Health.
} 


\section{Introduction}

This study was grounded in a mixed methods feasibility trial, which sought to reduce sedentary behaviour (SB) among older adult patients with Chronic obstructive pulmonary disease (COPD). COPD is a lung ailment that is typically characterised by severe shortness of breath, chronic coughing, wheezing, purulent sputum production and fatigue (Han, Dransfield, and Martinec, Fernando 2017). Individuals with COPD have been observed to avoid physical activity and spend the majority of their time sedentary ( Matei et al., 2015, p2; Pitta et al., 2005; Polkey, 2011). It has been reported that physical inactivity puts patients at an increased risk of being admitted to hospital (Garcia-Aymerich et al. 2006), whereas replacing SB with low-intensity activity has been associated with reduced risk of an acute exacerbation (Donaire-Gonzalez et al. 2015) or a worsening of symptoms (Celli and Barnes 2007).

Our study was informed by observations that attempts to improve COPD patients' lifestyleembedded activity have had little success (Watz et al. 2014; Cindy Ng et al. 2012). Our impetus, thus, was that reducing SB might provide a more gentle way of improving these patients' health (Cavalheri et al. 2016; Hill et al. 2015).

Prior to the intervention we conducted qualitative interviews with the participants, asking them a free association question of what sitting meant to them. We found that the majority associated sitting with a rich variety of positive meanings. In trying to make sense of this finding we consulted an emerging literature that has found that sitting or sedentary behaviour can, indeed, also have beneficial health effects. For example, a consensus project has sought to categorise different types of sedentary behaviour and discern diverse social, psychological and environmental factors that affect it (Chastin, Schwarz, and Skelton, 2013). We consider that attempts to come up with a more contextual approach to health and to distinguish 
between beneficial and harmful sitting points a way forward from the one-dimensional or healthyist position that sees all inactivity as intrinsically negative. However, at the same time these attempts to categorise sitting are not necessarily reflexive about the values embedded in these categories. We note that the methodological positions adopted by scholars studying sedentary behaviour in public health contexts, both quantitative and qualitative, are conducive of focusing on physical or mental health inducing or "salutogenic" (Kikuchi et al. 2014) effects. We contend that the context of debilitating and progressive chronic disease reveals the focus on health benefits as problematic, as it downplays other values, such as enjoyment of life. Further, we contend that doing qualitative research within a trial is inherently contradictory, as trials are, by definition, driven by a goal, such as behaviour change. The critically reflexive qualitative research that we adopted, on the contrary, sought to open up and question goals and categories if needed.

This paper then makes two arguments and contributions to discussions on sedentary behaviour. First, it critically reflects on the methodologies underpinned by specific values in research on sedentary behaviour - even when they try to understand why sitting may be beneficial — and argues for a qualitative research that would critically reflect on those values and categories. Second, it presents findings of our research with older adults with COPD who associated sitting with laziness, ability, enjoyment and sadness - and what this might teach us about attempts to reduce sitting and specifically about the importance to appreciate other values besides health, such as enjoyment of life, which may be particularly important in the context of progressive chronic disease. In what follows we will first discuss the literature on sedentary behaviour from a critical methodological angle and then move on to describing our methods and discussing our findings. 


\section{Classifying sedentary behaviour}

Research on sedentary behaviour, for our methodological purposes, can be divided into roughly three approaches. First, quantitative research has sought to establish how sedentary behaviour is associated with adverse health outcomes and how certain categories of sedentary behaviour (e.g. watching television) have more significant negative effects than others. Second, quantitative research has sought to establish whether some types of sedentary behaviour may have not only negative but also positive effects on, for example, mood. Third, qualitative research has focused on barriers and facilitators to reducing sedentary behaviour as well as individuals' perceptions and experiences of sitting.

An example of the first kind of quantitative research is a recent systematic review that demonstrated that SB is associated with harmful effects on biomarkers for cardiovascular disease and metabolic syndrome, especially for people aged over 60 years (Wirth et al. 2016). Hamer \& Stamatakis (2014) examined the association between popular sedentary behaviour activities (TV viewing, internet use, reading) and mental health. They did not find an increase in depressive symptoms from baseline to follow-up, but a longer period of TV watching was associated with higher depressive symptoms. Internet browsing and reading were associated with lower depressive symptoms, thereby suggesting that different sedentary activities may have different impacts on mental health.

The second type of quantitative research focuses specifically on differences between different types of sedentary behaviour. O’Neil \& Dogra, (2015) observed that especially sedentary activities that were social or provided cognitive stimuli, such as computer use or doing crosswords, were associated with self-reported psychosocial well-being in middle-aged and older adults, such as mood. Similarly, Kikuchi et al. (2014) found that what was defined as active sedentary activities, such as reading, were associated with higher physical activity 
(PA) levels, whereas passive activities, such as watching TV, were associated with lower PA levels.

Qualitative studies have typically explored barriers and facilitators to reducing SB. Chastin et al., (2014) found that many older women considered sedentary activities, such as playing bingo or reading, as pleasurable and many did not see the benefits of standing more (Chastin et al. 2014). A qualitative study exploring barriers and facilitators to reducing sedentary behaviour among overweight and obese older adults after an intervention (a wearable sitting time monitor) also found that barriers included enjoying sedentary activities or being too ill. Participants were motivated to reduce their sitting to improve health, when they could do things they enjoyed, such as daily walking, or things that were easy to do in the physical environment, such as going up stairs (Greenwood-Hickman, Renz, and Rosenberg 2016).

In a focus group study Mcewan et al., (2016) found that older adults considered sitting to have cognitive, social and psychological benefits, for example, by keeping their minds active (when reading), by sitting and socialising with friends and by filling their time doing things such as watching television (Mcewan, Tam-Seto, and Dogra 2016). Pertinent to our study on individuals with chronic disease an interview study examined the experiences of sitting of those living with rheumatoid arthritis (RA) (Thomsen et al. 2015). They found that participants were struggling between good and bad days and had to adapt to sitting down when they were tired or experienced pain, although some participants noted that their sitting had nothing to do with their RA but was a personal preference (pp.5-6).

Overall, these studies have observed that sedentary behaviour can have measurable beneficial effects on physical or mental health and that in qualitative studies participants often perceive sedentary activities as positive or beneficial. However, this situation presents a conundrum, as experiencing sedentary behaviour as positive creates a barrier to reducing it. Scholars have 
sought to overcome this contradiction by, for example, suggesting making sedentary social activities more active (O’Neil \& Dogra, 2015). We suggest, that fundamentally the contradiction reveals that there are multiple values or goods associated with sedentary behaviour besides health, such as enjoyment of life. We further contend that values, such as enjoyment of life, become particularly pertinent when studying individuals with progressive chronic disease, as it underlines the intrinsic importance of enjoying life in a situation where it becomes increasingly difficult.

\section{The special case of COPD}

To understand sedentary behaviour against the context of COPD it is useful to briefly review qualitative studies on the condition. Research has found that individuals with COPD often struggle to perform daily activities, such as walking or housework, and they often feel too tired to perform them and need to rest in between (Paap et al. 2014). Barnett (2005) reported that individuals with COPD considered caring for the self (washing, dressing) as difficult but that trying to perform them was important to preserve a sense of normality and independence (Barnett 2005). Harris, Hayter, \& Allender (2008) observed that whilst COPD restricted participants' ability, they tried to remain physically active within their own boundaries and capabilities as this gave them a sense of accomplishment (p707)(Harris, Hayter, and Allender 2008) . Thorpe, Kumar and Johnston's (Thorpe, Kumar, and Johnston 2014) identified barriers to physical activity (PA) among individuals with COPD and found that the self (including age and oxygen therapy), health (comorbidities, COPD and physical health), and the environment (personal surrounding, finances and access to transport) impeded individuals from engaging in PA. Dobbels et al., (2014) also found that for COPD patients PA meant doing small amounts of walking, household tasks, self-care (dressing, cleaning), walking up 
stairs and doing leisure activities, and that patients took breaks, paced themselves, used aids or gave up performing some activities in order to cope with their symptoms.

There have also been more general studies on patients with COPD. Marx et al., (2016) found that lack of mobility affected participants' normal daily activities, which eventually led to social isolation, fewer life opportunities and reduced independence leaving them feeling 'at the mercy of the disease' and their attempts to keep a sense of 'normality' became impossible (p3). Pinnock et al (2011) interviewed COPD patients over a period of 18 months to understand their experience as the illness progressed (Pinnock et al. 2011). The interviews described accepting the disruptive nature of the illness as 'a way of life' forming a 'chaos narrative' whereby activities and routines became increasingly challenging, even if many patients also normalised their illness as 'a result of old age' (p7).

As highlighted by these studies, COPD adds another dimension to sedentary behaviour. Individuals with COPD may find performing normal daily activities, such as chores and selfcare, difficult, these routines becoming a major part of their PA and needing to be paced to accommodate symptoms. The progressive illness also manifests itself in a loss of a sense of normality and a sense of chaos or being at the illness' mercy.

\section{Methods}

\section{Recruitment and participants}

Our qualitative study was nested within a feasibility trial seeking to reduce sedentary behaviour in COPD patients, who had been hospitalised following an acute exacerbation (for the protocol see (Orme et al, 2016) The study was conducted at a hospital in the UK Midlands between February and June 2016. Patients had to be between 40 and 85 and have a 
confirmed diagnosis of COPD, with this being their primary reason for their admission to hospital. Patients were invited to take part during their hospital stay by a study researcher and randomised into two interventions and one control arm, both of the intervention arms focused on reducing sedentary behaviour. In total, 111 patients were approached, and a total of 35 patients consented to be part of the study. The majority of patients were female (15 of 33) and over the age of 60 (15). Three patients in the sample were on home oxygen.

We interviewed the 21 participants, who agreed to take part and were randomised into one of the two intervention arms. The participants were interviewed before the intervention was introduced.

The aim of the trial was to assess the feasibility and acceptability of using an educational booklet and a self-tracking device in individuals with COPD to reduce sedentary behaviour. The education group received written and verbal information about reducing sitting, such as top tips to sit less. The feedback and education groups were additionally given a self-tracking device to be worn around the waist (LUMO). The participants were also invited for two interviews, before and after the study. This article focuses on the first interviews.

\section{Interviews}

The interviews were conducted by a trained qualitative researcher at the hospital. An interview guide was developed collaboratively amongst the research team. The patients were asked to describe their daily lives, good days and bad days, physical activity, sitting and how they managed their COPD; they were also asked a free association question of what came to their mind when mentioned the word 'sitting' (Holloway and Jefferson 2000). Interviews lasted between 10 and 70 minutes, the average being 36 minutes. 
The mean age of the participants was 67 years (ranging 52-81 years). Fifteen of the participants were female and six were male; five of the participants were on home oxygen (a proxy for the severity of their COPD). Table 1 details the characteristics of the participants.

Table 1: Characteristics of participants

\begin{tabular}{|l|l|}
\hline Characteristic & Frequency \\
\hline Gender & 6 \\
Female & 15 \\
\hline Age (years) & \\
$51-60$ & 6 \\
$61-70$ & 5 \\
$71+$ & 10 \\
\hline Home oxygen use & 3 \\
Yes & 18 \\
No & \\
\hline
\end{tabular}

\section{Analysis of Interviews}

All interviews were audio-recorded and transcribed. Interviews were analysed thematically, and a constant comparative method was used to compare new codes with existing codes, and to investigate new codes against all data extracts (Glaser 2008; Glaser and Strauss 2009). Interview data was facilitated by Nvivo 10 qualitative software. Initial coding was done by $\mathrm{AB}$, in conversation with $\mathrm{BB}$ and $\mathrm{CC}$, a selection of interviews was inter-rater checked by $\mathrm{BB}$ and CC (Armstrong et al. 1997)(Denzin, N 1978), and the whole team discussed emerging 
themes. Our analysis focused on 'meanings' patients associated with sitting (Wilkinson 1998).

The analysis followed the basic principles of thematic analysis as outlined by Braun \& Clarke (2006), beginning with open coding, which were then distilled into thematic codes. The main focus in the first interviews were meanings and experiences of sitting, triggered by the free association question and follow-up questions. Four recurring themes were identified: (i) participants underlining they were not just sitting, (ii) experiencing sitting as enabling one to do activities, (iii) experiencing sitting as enjoyable or (iv) experiencing sitting as a sad state, being unable to do anything else and looking mournfully into past active life. The four themes will be discussed in more detail in what follows.

\section{Results}

\section{Not just sitting}

When asked to describe their sedentary behaviour, most of our participants emphasised that they did not just sit, instead they did something meaningful to them whilst sitting down;

I'm not one for sitting down, and I can't sit down and do nothing and that's why I read, I can't just sit, I have to be reading or something (Gladis, female)

I'm not sitting too long... When I'm in the conservatory, I'll stand in there watching the TV. Especially to give your legs a rest, or some exercise. I don't like sitting all day like some folks do (Rick, Male)

Sometimes I have a little nap on the chair, but I need that cause I'm up early, and I don't sleep very well cause my husband has osteitis, and he's tossing and turning and 
up at the toilet all night. So what sleep I can catch up on, I do when I've done my bits. It's not because Im lazy, but I just have to catch up so that doesn't really count (Joyce, female)

In these excerpts, similar to Mcewan et al (2016) study, our participants associated sitting with negative connotations, such as 'doing nothing' and being 'lazy.' These descriptions highlighted the stigma associated with sitting, as something opposed to being entrepreneurial and active. Our participants did not associate their own sitting with these labels, seeing themselves as different, thereby deflecting the moral blame associated with sitting as potentially a sign of laziness or being unhealthy. This tendency to deflect blame by downplaying activities associated with unhealthy lifestyle has been observed by others studying sedentary behaviour (Greenwood-Hickman, Renz, and Rosenberg 2016).

However, most importantly the theme of not just sitting underlined the fact that people with COPD saw themselves doing activities that were meaningful and important to them whilst sitting. Thus, for them the important point about their behaviour was not sitting itself but what they were doing whilst sitting, which may be overlooked if one focuses on negative health consequences of SB.

\section{Sitting as enabling}

Many of our participants explained that since their diagnosis of COPD daily activities had become difficult, but that completing daily tasks allowed them to live a normal life. These activities included household chores, such as cooking, and self-care routines, such as dressing or washing one's self. Our participants explained that in order to enable them to complete 
such activities they needed to sit and catch their breath or rest for a few minutes to be able to continue the task, including self-care and walking:

To shower I have a perch to use. When preparing dinner I have to sit, and my husband dishes up whilst I get my breath back (Iris, female)

I can't do it all without a break. I won't go anywhere, unless I know there are some benches for me to sit down and get my breath back. I get scared of doing stuff, if I don't have a seat around me, because I have my limits (Fran, Female)

Participants discussed sitting in terms of enabling them to do activities with reference to household chores. This was more common among female participants, which underlines the gendered nature of housework.

Sometimes I just stay in and tinkle about doing my ironing in stages, perhaps iron a shirt and sit down (Kate, female)

I try to keep myself active doing things: moving around all day, cleaning, walking, fetching papers, picking up after my husband, washing. Just general house stuff, the things you have to do cause nobody else is going to do it for you. Well not in my case (Joyce, Female)

Men did not describe doing household chores but also associated sitting with pacing an activity, such as walking a dog:

When I walk the dog, I don’t rush I walk stop, slow down, walk, sit down, walk, sit I just take my time. Sometimes I sit on a wall for a minute, then I carry on (Steve, male). 
Both female and male participants living alone and participants who lived with partners or had carers discussed sitting to take a break to allow them to do things by themselves, giving them a sense of independence:

I get myself dressed and showered, but it does take me a long time, and I have a seat to help me. It's the only way I can do it myself. I step into the shower, and then I've got this stool for when I come out, so I sit down and get my breathe back and wrap the towel around me, gradually get myself dry. It takes me ages to get dressed, but I do it myself (Kate, female)

I do sit a lot to help me, cause I mean my parents are too old to look after me. I've got my little dog for company, but I'm on my own, so I've had to learn to do things by myself (Helen, female)

Sometimes enabling sitting was referred to in more general terms as catching a breath to be able to continue with usual activities:

I stop doing what I'm doing and basically just take a chill pill, sit and just try and take some deep breaths (Helen, female)

I have to slow my own breathing down, so I do end up sitting down and resting a lot, but I have to don't I (Valarie, female)

Thus, sitting for the participants with COPD was not associated with being physically inactive but enabling them to be active by taking breaks in between activities from taking a shower to walking the dogs. This type of pacing activities with breaks has been reported in several studies on COPD patients (Dobbels et al. 2014; Pinnock et al. 2011). From the point of view of efforts to reduce sedentary behaviour it is important to pay attention to this way of approaching sitting among individuals with chronic diseases, associated with fatigue and 
breathlessness, as any effort to reduce SB that interferes with pacing activities e.g. reduce rest periods could be experienced as disruptive. In this situation sedentary behaviour may even be a misnomer as it refers to resting to be able to perform activities. However, light physical activity is not the only or necessarily the most important issue at stake here. Being able to perform activities by resting at intervals also enabled our participants to continue taking care of themselves and their households, which helped them to live independently or with partners and gave them a sense of normality and accomplishment. Thus, sitting for these adults with chronic disease actually formed part of activity, enabling it; this activity being associated with benefits far beyond physical health, such as independence and sense of being able to live a normal life.

\section{Sitting as enjoyable}

Many of our participants also associated sitting with positive meanings; it was experienced as enjoyable in different ways.

Many participants associated sitting with relaxation, but with an emphasis on a need to recover or rest, similar to the previous theme of sitting as enabling. However, relaxing sitting could be described in elaborate terms of napping, reading the paper and snuggling with the dog, all of which were associated with the need to recharge and with pleasure or enjoyment:

In the afternoon I do get to the stage where I sleep for probably 20 minutes, maybe 40 minutes. I have a cat nap, I'm not asleep, I know what's going on. I'll lay on the seat and bring the chair up and the dog will jump up here and he'll go sleep on my armchair. I'll have a nod or might read the paper s and all you're doing is charging your batteries up (Steve, male). 
Sitting was also often associated with simple enjoyable activities, such as watching soap operas, reading a book or going for lunch with friends:

I'm relaxed, totally relaxed. I don't get hecked up, it's safe. I quite enjoy sitting because it takes my mind of things (Kate, female)

Sitting to me is just sitting in my chair watching my telly for about $10-15$ minutes. ... I do like watching my soaps (Joyce, female)

I go out for lunch with friends and that's sitting. I'll sit in the garden maybe with a book. I sit for the sake of it, sit for any reason really. I's not because I'm on my feet all day, I just enjoy it (Erica, female)

Others enjoyed sedentary activities that have been noted to be more mentally active or have cognitive benefits (refs), such as reading, and the participants also saw these activities as keeping their minds active:

I'm not really one for sitting down and doing nothing and that's why I read it's better than doing nothing but it's all I can do now really. Knit and sew, anything to keep my brain ticking really (Gladis, female)

I could sit with a good book for hours and I do frequently. And my painting I do that a lot and that's what I do most the time really. I find both really relaxing and I enjoy it but I can't just sit there and do nothing, I have to be doing something like that to keep my mind ticking you see, something to concentrate on (Gary, male)

A couple of the male participants discussed activities they enjoyed, noting that although they sat doing them, they were still being physically active, as also observed by Mcewan et al., (2016): 
I just go in the garage and build up dolls' houses and stuff. I'm standing up or sitting down, but I'm moving all the time. I'm not just sitting there unless it's something small and even then its exercise isn't it for the upper body (Steve, male)

I go fishing once a week and when it's nice I go every weekend too. I go on a couple of socials too with the guys who are part of the club, few cans of beer. It's not sitting, because it's exercise to fish, it can be hard work! (Rick, male)

The sedentary activities that our participants enjoyed have been categorised as active (reading, painting) or passive (watching television), social (having lunch with friends) or perhaps as incorporating light physical activity (fishing). Our participants, just like previous research in this area (Mcewan et al., 2016) saw these activities as having benefits in terms of maintaining cognitive ability or "mind ticking," which in the context of memory loss in old age can be framed as a health benefit. Similarly, socialising can be seen to have mental health benefits in terms of breaking isolation for everyone but, perhaps especially for older adults and those chronically ill. Also, hauling fishing equipment might be seen as light physical activity and therefore as having health benefits.

However, the question remains whether watching soap operas on television should account as having health benefits? Kikuchi et al., (2014) have categorised television watching as particularly detrimental or passive form of sitting in terms of being negatively associated with PA and mental health, as opposed to active forms of sitting, such as using a computer. These associations are interesting. However, for a communication scholar the distinctions between active and passive sitting vis a vis media use connects to a long-standing scholarship in this area filled with problems. In the early $20^{\text {th }}$ century the Frankfurt School Marxists distinguished between popular music, such as jazz, seen as pacifying mass culture, as opposed to classical music enhancing critical thinking (Adorno, 2001). 
Later scholarship criticised these distinctions for fairly obvious class, gender and race based biases (Huyssen, 1986).

So, the question remains to what extent these categories reflect old associations between television and passive low or mass culture, seen as requiring less mental processing, which have criticised for being shot through with classist, sexist and racist underpinnings.

Being reflective of the categories we use or are take for granted is one of the key goals of qualitative research that seeks to be open to new vistas and foreground neglected or silenced experiences and realities (Lather, 1993). Therefore, rather than classify our participants' television viewing merely as a barrier to reducing sedentary behaviour, we suggest taking our participants comments at face value and appreciate that it is intrinsically enjoyable. So, we contend that television watching in this instance need not be evaluated vis a vis health, as having potentially negative consequences or as potentially having positive health or mental health consequences, such as mood or sense of belonging (O’Neil \& Dogra, 2015). However, rather than subsume all values under one master value ('health') we should be open to multiplicity of values that may be contradictory (Lather, 1993). So, our participants' experience of enjoying television watching should be considered just that, as articulating the intrinsic value of enjoyment in life, which does not necessarily have to have anything to do with health, even if it may. It may also be that the value of enjoyment of life is particularly pertinent in the context of chronic progressive disease, as will be discussed next.

\section{Mournful sitting}

Some of our participants felt that their COPD had taken over or overwhelmed their lives. For these participants sitting became a way of life, which they did not associate with positive 
meanings. They felt they 'had to' sit, as otherwise they would get too breathless or tired, and often mourned a previous, active and happy life:

What was a happy life at one time becomes a sad life because you're stuck in that bloody arm chair and your arm chair becomes your safe guard as it were (Patrick, male)

Some days I just dread getting off the settee because while I'm sat there I'm breathing fine. When I've gotta get up to do something, well I have to build me selfup for it (Valarie, female)

Many participants discussed feelings of resignation:

I sit and watch telly most of the time, cuz at the moment; I just can't be bothered, can't be bothered to get ready and can't be bothered to make an effort anymore. I've sort of given up. So I just sit and watch the world go by (Glenda, female)

My whole life has changed. Everything's just gone out the window. I've got nothing left (Valarie, female)

Some participants discussed feelings of isolation and a general resentment about their illness, which prevents them for living a fulfilled life:

It's a very lonely disease, because you can't keep up with friends, it's impossible. Sometimes it's impossible to walk, it separates you from your family, because I hold them back and so you miss out on a great deal in life. It can become very solitary (Patrick, male)

All participants using home oxygen discussed how being on oxygen made them give up on many aspects of their life: 
I'm lucky if I can get out my armchair and get to the loo and back... I can't cook cause it's difficult with an oxygen tubes hanging out your nose. What else can I do but sit in my armchair? Sometimes I sleep there at night, because it's near the loo and I worry about moving. Sitting is relief to me because, I can't fall over, I won't turn dizzy, and I'm just safe and resting. I don't particularly enjoy it, it's just a necessity (Ann, female)

These participants often experienced feelings of sadness and resignation associated with their COPD. They expressed fear of getting up and standing, and often talked about waiting to die, or it not mattering if they did. They had lost a lot of their independence and were unable to do things they used to do. As such the participants felt resigned to their COPD and sitting was the only thing they felt they could do, even if it was not perceived as positive. Whilst some of these participants also engaged in activities whilst sitting, for example watching television, they discussed these activities and sitting in more negative terms as opposed to the participants who spoke about enjoying those activities. Thus, for these participants sitting was not enjoyable, it was a predicament bestowed upon them by their illness, as they were not capable of doing other things or basically to enjoy life anymore. A significant feature of these interviews was a tendency to look back on good, active life, which is why we termed them mournful sitters, as their time horizon was focused on the past which they yearned for, feeling sad about not being able to take part in activities with family and friends and feeling they were a burden to them.

Studies on sedentary behaviour have observed that feeling tired or ill is a barrier to reducing SB among older adults (Chastin et al. 2014; Greenwood-Hickman, Renz, and Rosenberg 2016) and those suffering from arthritis (Thomsen et al. 2015). Furthermore, being too ill and breathless was also a barrier for PA among COPD patients (Paap et al. 2014). 
Furthermore, the experiences of loss and isolation (Ek \& Ternestedt 2008), of limited opportunities, particularly when on oxygen (Marx et al 2016)) and being resigned to their condition (Ek and Ternestedt 2008) (Marx et al. 2016) reported in the literature on COPD patients, resonate with our findings.

The participants who associated their sitting with sadness and mournfulness were more severely ill than the others, all the three participants on oxygen discussed sitting in terms of sadness. One could simply say that being too ill is a barrier to reducing SB in this context, which is true. However, there is much more to our interviews with these people than this. In the context of the end stages of progressive chronic illness, such as COPD, framing sitting as a choice, which is often the presumption in behaviour change interventions, is not appropriate. Our participants did not experience their sitting as a choice, rather they underlined how they would rather be active but were confined to their chair due to breathlessness and inability to stand up, even sleeping on the chair during nights for fear of not being able to get up from bed. Further, the severely ill participants did not discuss sedentary activities, including television watching, in terms of enjoyment. Rather they considered them the only thing they could do, a predicament rather than pleasure. Against this we contend that the enjoyment attached to sedentary activities should be understood as an intrinsic value in and of itself, rather than as a derivative of health effects, either negative or positive. The importance of being able to enjoy life, or joie de vivre, comes into particularly stark relief in the context of progressive chronic disease, where patients increasingly become unable to enjoy life or consider their lives worth living. 


\section{Discussion}

Health is increasingly understood to encompass not just physical health or the absence of disease but a broader state of "physical, mental and social well-being" (WHO, 2018). The recent discussions on sedentary behaviour and its potential beneficial effects embrace this broader definition and are moving research in this area to a more nuanced direction, away from the simplistic "move more and sit less" messages (Phoenix \& Bell, 2018).

Quantitative and qualitative research on sedentary behaviour has reported that sedentary activities, such as socialising, media use and hobbies, can benefit the psychosocial wellbeing of older adults and be associated with better mood, sense of belonging and perceived as enjoyable (Greenwood-Hickman, Renz, and Rosenberg 2016; O’Neil \& Dogra, 2015). Research on barriers to reducing sedentary behaviour or increasing light physical activity include feeling ill, pain, infrastructural issues, such as transport, and enjoying sedentary activities (Chastin et al., 2014; Greenwood-Hickman, Renz, and Rosenberg 2016).

These are all valuable observations but keep coming back to the question of how to acknowledge benefits of sedentary activities and that enjoying these activities is one of the major barriers to reduce sedentary behaviour. Our study gives some answers to this question from the point of view of chronic disease, which highlights three issues: (i) individuals have different abilities to reduce sedentary behaviour, (ii) we should acknowledge the independent value of enjoying (sedentary) activities, rather than reduce them to health and (iii) mixed methods research in the context of trials runs into contradictions.

We will discuss these three points in what follows. But before proceeding we will encapsulate the findings of our trial to set the discussion in its context. The mixed methods feasibility trial had modest results in terms of recruitment and retention of participants. 
However, the small group who did complete the intervention reduced their sedentary time. The qualitative follow-up interviews revealed that participants stood up when it fitted with the flow of their everyday activities but refused to stand up when the self-monitor prompted them, if it interrupted activities perceived as important and enjoyable, such as watching an interesting television programme or enjoying a catnap with pets. The most severely ill participants, which in the initial interview associated sitting with sadness all dropped out of the study citing ill health (Orme et al, 2018).

Against this background our findings confound the general premise underpinning behaviour change interventions that reducing sedentary behaviour is always a choice, related to factors, such as knowledge, motivations and preferences. It has been noted that pain and disability can hinder ability to exercise and should not be downplayed (Williams et al, 2017), similarly it has been noted that barriers to PA among COPD patients need addressing (Thorpe, Kumar, and Johnston 2014). In our study participants who sat in order to enable activity highlight the importance to consider the abilities and limits of participants. In the case of these participants, attempts to reduce sedentary behaviour would need to respect participants' pacing and any interventions in this respect would be well advised to consider resting between activities not as a barrier but as a facilitator. Participants associating sitting with sadness is a case in point, as they simply felt they could not stand more due to their illness. One of the inclusion criteria for our trial was an assessment by a COPD specialist nurse that the patient was capable of light PA, however, the life world experience of our participants indicated that they felt too ill, too sad and struggled too much with everyday life and their emotions to engage with selfmonitoring or education. Based on our observations an altogether different intervention may have been needed to improve the quality of life of these participants. Further, as also pointed out by Williams et al (2017) vis a vis individuals with arthritis and spinal cord injury, attempts to increase activity need to take care not to induce a sense of personal failure in 
vulnerable participants, which may be especially an issue with individualistic interventions, such as self-monitoring.

Further, the value of enjoying sedentary activities, or any activities for that matter, came to particularly sharp relief in contrast with the experience of our participants who no longer enjoyed activities, feeling they were simply forced to sit by their illness. So, the fairly ordinary descriptions of enjoying watching soap operas or building doll's house illustrate the importance of pleasure, of being able to enjoy life, which the most ill and resigned COPD patients could no longer do. Therefore, rather than demonise activities that may seem the wrong choice, detrimental to health or inducing physical and mental passivity, one may need to appreciate their intrinsic value of offering enjoyment to any people but especially for those with life-limiting, progressive illnesses, who may not have so many sources of pleasure available to them. It may be that the idea of intruding upon these activities (one of the top tips was to get up at regular intervals whilst watching television) may not be appropriate, and it did not always work so well in our study. It could be that a better idea would be to start from the opposite end and rather than disrupt pleasurable activities ask the participants what they find pleasurable to find out a way to enhance pleasurable light activity. As has been noted (Williams et al, 2017) the issue may be that taking care of health is framed as work or a chore rather than as something that gives one enjoyment, which may miss one of the most potent activity inducing motivations of having fun. The reverse of that in our study would be to make sure that enjoyable activities are not framed as the problem.

The final observation of our study is that the mixed methods design within a randomised controlled trial (RCT) is particularly challenging. RCTs are predicated on a research driven goal, often articulating technological possibilities, needs of the healthcare system and prevailing social ethos, such as changing behaviour to reduce sedentary time. In this framework qualitative research if often delegated to secondary role (Hesse-Biber, 2010), to 
find out processes that may enhance or impede participants' engagement with the intervention. This design goes against the basic principles of qualitative research, which is to generate rather than test hypotheses. In particular, the RCT design goes against the grain of interpretivist and post-structuralist or post-positivist approaches in qualitative research, which seek to foreground the standpoints of often silenced groups, such as patients, and to be willing to disrupt achieved wisdom and categories (Lather, 1993, Saukko, 2003). Whilst we have learnt a great deal both substantively and methodologically by engaging with a feasibility trial, the qualitative researchers also found it difficult to fit the findings into the format of reporting trial results, where viewpoints that do not support the premises of the trial always seem a bit of an embarrassment. It could be that a different design would be more feasible for a more egalitarian collaboration between qualitative and quantitative research. One possibility could be fronting interventions with a coproduction phase where the potential participants or users, including patients and staff, could co-create ideas to improve the problem at hand, such as the health and wellbeing of COPD patients.

\section{Decleration of interest}

No potential conflict of interest was reported by the authors. 


\section{References}

Adorno, Theodor. 2001. "The culture industry: selected essays on mass culture. London: Routledge. doi: 10.4324/9780203996065

Armstrong, David, Ann Gosling, John Weinman, and Theresa Marteau. 1997. "The Place of Inter-Rater Reliability in Qualitative Research: An Empirical Study.” Sociology 31 (3). CAMBRIDGE UNIVERSITY PRESS: 597-606. doi:10.1177/0038038597031003015.

Barnett, Margaret. 2005. “Chronic Obstructive Pulmonary Disease: A Phenomenological Study of Patients' Experiences.” Journal of Clinical Nursing 14 (7): 805-12. doi:10.1111/j.1365-2702.2005.01125.x.

Braun, Virginia, and Victoria Clarke. 2006. "Using Thematic Analysis in Psychology" 3: 77101. doi:10.1191/1478088706qp063oa.

Cavalheri, Vinicius, Leon Straker, Daniel F. Gucciardi, Paul A. Gardiner, and Kylie Hill. 2016. "Changing Physical Activity and Sedentary Behaviour in People with COPD." Respirology 21 (3): 419-26. doi:10.1111/resp.12680.

Celli, B. R., and P. J. Barnes. 2007. "Exacerbations of Chronic Obstructive Pulmonary Disease.” European Respiratory Journal 29 (6): 1224-38. doi:10.1183/09031936.00109906.

Chastin, Sebastien, Nicole Fitzpatrick, Michelle Andrews, and Natalie DiCroce. 2014. "Determinants of Sedentary Behavior, Motivation, Barriers and Strategies to Reduce Sitting Time in Older Women: A Qualitative Investigation.” International Journal of Environmental Research and Public Health 11 (1). Multidisciplinary Digital Publishing Institute: 773-91. doi:10.3390/ijerph110100773. 
Chastin, Sebastien Francois Martin, Ulf Schwarz, and Dawn Ann Skelton. 2013.

"Development of a Consensus Taxonomy of Sedentary Behaviors (SIT): Report of

Delphi Round 1." Edited by Deborah Kado. PLoS ONE 8 (12). Public Library of

Science: e82313. doi:10.1371/journal.pone.0082313.

Cindy Ng, Li Whye, Jenny Mackney, Sue Jenkins, and Kylie Hill. 2012. “Does Exercise Training Change Physical Activity in People with COPD? A Systematic Review and Meta-Analysis." Chronic Respiratory Disease 9 (1): 17-26.

doi:10.1177/1479972311430335.

Denzin, N, K. 1978. The Research Act: A Theoretical Introduction to Sociological Methods. New York: McGraw-Hill.

Dobbels, F., C. de Jong, E. Drost, J. Elberse, C. Feridou, L. Jacobs, R. Rabinovich, et al. 2014. "The PROactive Innovative Conceptual Framework on Physical Activity." European Respiratory Journal 44 (5): 1223-33. doi:10.1183/09031936.00004814.

Donaire-Gonzalez, David, Elena Gimeno-Santos, Eva Balcells, Jordi de Batlle, Maria A. Ramon, Esther Rodriguez, Eva Farrero, et al. 2015. "Benefits of Physical Activity on COPD Hospitalisation Depend on Intensity.” European Respiratory Journal 46 (5): 1281-89. doi:10.1183/13993003.01699-2014.

Ek, Kristina, and Britt-Marie Ternestedt. 2008. "Living with Chronic Obstructive Pulmonary Disease at the End of Life: A Phenomenological Study." Journal of Advanced Nursing 62 (4). Blackwell Publishing Ltd: 470-78. doi:10.1111/j.1365-2648.2008.04611.x.

Garcia-Aymerich, J, P Lange, M Benet, P Schnohr, and J M Anto. 2006. "Regular Physical Activity Reduces Hospital Admission and Mortality in Chronic Obstructive Pulmonary Disease: A Population Based Cohort Study." Thorax 61 (9): 772-78. 
doi:10.1136/thx.2006.060145.

Glaser, Barney G. 2008. "The Constant Comparative Method of Qualitative Analysis.” Grounded Theory Review 7 (3): 436-45.

Glaser, Barney G., and Anselm Leonard Strauss. 2009. The Discovery of Grounded Strategies for Qualitative Research. New York: Aldine de Gruyter. https://books.google.com/books?hl=en\&lr=\&id=rtiNK68Xt08C\&pgis=1.

Greenwood-Hickman, Mikael Anne, Anne Renz, and Dori E. Rosenberg. 2016. "Motivators and Barriers to Reducing Sedentary Behavior among Overweight and Obese Older Adults." Gerontologist 56 (4). Oxford University Press: 660-68. doi:10.1093/geront/gnu163.

Hamer, Mark, and Emmanuel Stamatakis. 2014. "Prospective Study of Sedentary Behavior, Risk of Depression, and Cognitive Impairment.” Medicine and Science in Sports and Exercise 46 (4): 718-23. doi:10.1249/MSS.0000000000000156.

Han, MeiLan King, Mark T Dransfield, and J Martinec, Fernando. 2017. “Chronic Obstructive Pulmonary Disease: Definition, Clinical Manifestations, Diagnosis, and Staging." UpToDate. https://www.uptodate.com/contents/chronic-obstructivepulmonary-disease-definition-clinical-manifestations-diagnosis-and-staging.

Harris, David, Mark Hayter, and Steven Allender. 2008. "Improving the Uptake of Pulmonary Rehabilitation in Patients with COPD: Qualitative Study of Experiences and Attitudes." The British Journal of General Practice : The Journal of the Royal College of General Practitioners 58 (555): 703-10. doi:10.3399/bjgp08X342363.

Hesse-Biber, Sharlene. 2010. "Qualitative approaches to mixed methods practise" Qualitative enquiry 16 (6): 455-468. doi:10.3399/bjgp08X342363. 
Hill, K., P. A. Gardiner, V. Cavalheri, S. C. Jenkins, and G. N. Healy. 2015. "Physical Activity and Sedentary Behaviour: Applying Lessons to Chronic Obstructive Pulmonary Disease.” Internal Medicine Journal 45 (5): 474-82. doi:10.1111/imj.12570.

Holloway, Wendy, and Tony Jefferson. 2000. Doing Qualitative Research Differently: Free Association, Narrative and the Interview Method. London: Sage Publications. https://books.google.co.uk/books?hl=en\&lr=\&id=CFaC5TVKfM8C\&oi=fnd\&pg=PP11 $\& d q=$ free + association + method + in + psychology\&ots $=\mathrm{bDL} 12 \mathrm{oE} 5$ Sk\&sig $=$ ouMvRTtL $7 \mathrm{kr}$ SVlGBeyiASj4EEJw\#v=snippet\&q=free association $\& \mathrm{f}=$ false.

Huyssen, Andreas. 2003. "Mass culture as women". In Huyssen, Andreas (Ed.), After the great divide, modernism,mass culture, postmodernism (pp. 44-65). Bloomington: Indiana University Press Ltd.

Kikuchi, Hiroyuki, Shigeru Inoue, Takemi Sugiyama, Neville Owen, Koichiro Oka, Tomoki Nakaya, and Teruichi Shimomitsu. 2014. "Distinct Associations of Different Sedentary Behaviors with Health-Related Attributes among Older Adults." Preventive Medicine 67 (October): 335-39. doi:10.1016/j.ypmed.2014.08.011.

Lather, Patti. 1993. "Fertile obsession; validity and poststructuralism.” The sociological quartley 34 (4): 673-693. URL: https://www.jstor.org/stable/4121374

Marx, Gabriella, Maximilian Nasse, Henrikje Stanze, Sonja Owusu Boakye, Friedemann Nauck, and Nils Schneider. 2016. "Meaning of Living with Severe Chronic Obstructive Lung Disease : A Qualitative Study.” BMJ Open 6 (12): 1-8. doi:10.1136/bmjopen2016-011555.

Mcewan, Toshoya, Linna Tam-Seto, and Shilpa Dogra. 2016. "Perceptions of Sedentary Behavior Among Socially Engaged Older Adults.” The Gerontologist, February. Oxford 
University Press, gnv689. doi:10.1093/geront/gnv689.

O’Neill, Carley, and Shilpa Dogra. 2016. "Different Types of Sedentary Activities and Their Association with Perceived Health and Wellness among Middle-Aged and Older Adults: A Cross-Sectional Analysis.” American Journal of Health Promotion 30 (5): 314-22. doi: $10.1177 / 0890117116646334$.

Orme, Mark, Amie Weedon, Dale Esliger, Paula Saukko, Mike Morgan, Mike Steiner, John Downey, Sally Singh, and Lauren Sherar. 2016. "Study Protocol for Chronic Obstructive Pulmonary Disease-Sitting and ExacerbAtions Trial (COPD-SEAT): A Randomised Controlled Feasibility Trial of a Home-Based Self-Monitoring Sedentary Behaviour Intervention.” BMJ Open 6 (10): e013014. doi:10.1136/bmjopen-2016013014.

Orme, M, Weedon, A, Saukko, P, Esliger, D, Morgan, M, Steiner, M, Downey, J, Sherar, L, Singh, S (2018) Findings of a randomised controlled feasibility trial to reduce sedentary time using wearable and mobile technologies with educational support: Chronic Obstructive Pulmonary Disease-Sitting and ExacerbAtions Trial (COPD-SEAT), Journal of Medical Internet Research: mHealth and uHealth, 6(4), 84, ISSN: 2291-5222. DOI: 10.2196/mhealth.9398.

Paap, Muirne CS, Christina Bode, Lonneke IM Lenferink, Lianne C Groen, Caroline B Terwee, Sara Ahmed, Owis Eilayyan, and Job van der Palen. 2014. "Identifying Key Domains of Health-Related Quality of Life for Patients with Chronic Obstructive Pulmonary Disease: The Patient Perspective." Health and Quality of Life Outcomes 12 (1): 106. doi:10.1186/s12955-014-0106-3.

Phoenix, Cassandra, \& Bell, Sarah L. 2018. "Beyond "Move More": Feeling the Rhythms of physical activity in mid and later-life" Social Science and Medicine. http://doi.org/10.1016/j.socscimed.2018.05.006 
Pinnock, Hilary, Marilyn Kendall, Scott A Murray, Allison Worth, Pamela Levack, Mike Porter, William MacNee, and Aziz Sheikh. 2011. "Living and Dying with Severe Chronic Obstructive Pulmonary Disease: Multi-Perspective Longitudinal Qualitative Study." BMJ Supportive \& Palliative Care 1 (2): 174-83. doi:10.1136/bmjspcare.d142rep.

Polkey, Michael I. 2011. “Attacking the Disease Spiral in Chronic Obstructive Pulmonary Disease: An Update." Clinical Medicine, Journal of the Royal College of Physicians of London 11 (5): 461-64. http://www.ncbi.nlm.nih.gov/pubmed/16688981.

Saukko, Paula. 2003. "New ethnography and understanding the Other" In Paula. Saukko, Doing Research in Cultural Studies (pp. 55-73). London, United Kingdom: SAGE Publications Ltd. http://doi.org/10.4135/9781849209021

Thomsen, Tanja, Nina Beyer, Mette Aadahl, Merete L Hetland, Katrine Løppenthin, Julie Midtgaard, and Bente A Esbensen. 2015. "Sedentary Behaviour in Patients with Rheumatoid Arthritis: A Qualitative Study.” International Journal of Qualitative Studies on Health and Well-Being 10: 28578. http://www.ncbi.nlm.nih.gov/pubmed/26462971.

Thorpe, Olivia, Saravana Kumar, and Kylie Johnston. 2014. "Barriers to and Enablers of Physical Activity in Patients with COPD Following a Hospital Admission: A Qualitative Study.” International Journal of COPD 9 (January): 115-28. doi:10.2147/COPD.S54457.

Watz, Henrik, Fabio Pitta, Carolyn L. Rochester, Judith Garcia-Aymerich, Richard ZuWallack, Thierry Troosters, Anouk W. Vaes, et al. 2014. “An Official European Respiratory Society Statement on Physical Activity in COPD.” European Respiratory Journal 44 (6). http://erj.ersjournals.com/content/44/6/1521. 
WHO. (2018). “About us, constitution of WHO principles.” Journal of Health Psychology 3 (3): 329-48. doi:10.1177/135910539800300304. World Health Organisation Accessed: $8^{\text {th }}$ Dec 2018. URL: https://www.who.int/about/mission/en/

Wilkinson, Sue. 1998. "Focus Groups in Health Research.” Journal of Health Psychology 3 (3): 329-48. doi:10.1177/135910539800300304.

Williams. 1998. "Focus Groups in Health Research.” Journal of Health Psychology 3 (3): 329-48. doi:10.1177/135910539800300304.

Williams, Toni L, Hunt, Emily R, Papathomas, Anthony \& Smith, Brett. 2018. "Exercise is medicine? Most of the time for most; but not always for all". Qualitative Research in Sport, Exercise and Health, 10 (4): 441-456, doi: 10.1080/2159676X.2017.1405363

Wirth, K, J Klenk, S Brefka, and K Figuls. 2016. "Biomarkers Associated with Sedentary Behaviour in Older Adults: A Systematic Review.” doi:10.1016/j.arr.2016.12.002. 
Notes:

Abbreviations: COPD, chronic obstructive pulmonary disease; SB, sedentary behaviour. 